\title{
SMALL PET SCANNER \\ BASED ON MRI-COMPATIBLE LIGHT SENSOR
}

\author{
IJ. Molnar ${ }^{1}$, L. Balkay ${ }^{2}$ and E. Berenyi ${ }^{3}$ - DOI: 10.1051/epn/2015202 \\ ${ }^{1}$ Institute for Nuclear Research, Hungarian Academy of Sciences, Debrecen - HU \\ II ${ }^{2}$ Department of Nuclear Medicine, University of Debrecen - HU \\ ${ }^{3}$ Department of BioMedical Laboratory and Imaging Science, University of Debrecen - HU
}

Improving the quality of life of elderly people requires diagnostic and therapeutic capabilities for diseases of the central nervous system, such as Alzheimer's, Parkinson's, and epilepsy which have a rapidly growing impact on society. Minimallyinvasive imaging technologies such as PET and MRI allow for monitoring and tracking these illnesses, starting from their preliminary manifestations.

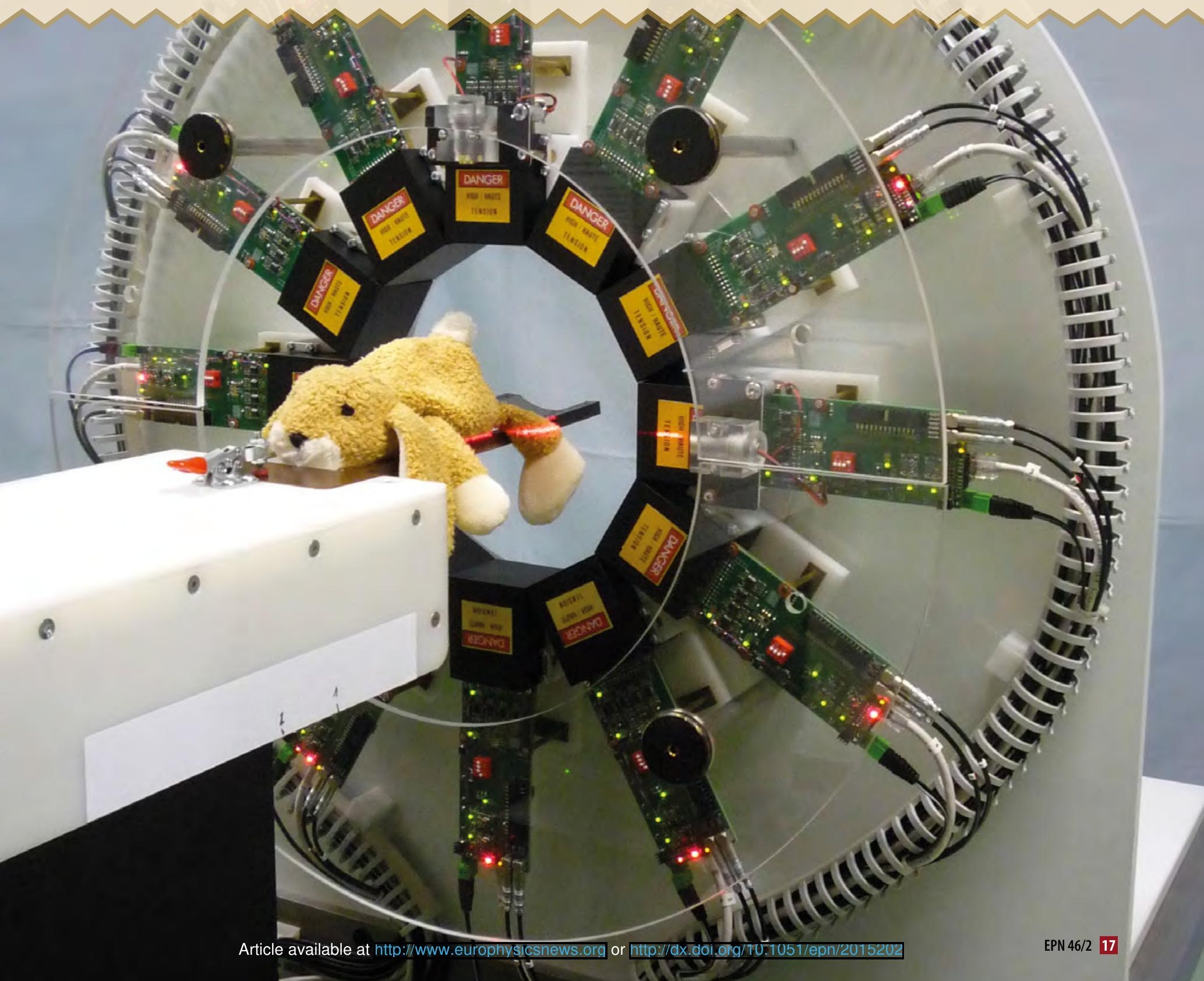


4 P.17: MiniPET-3 small animal PET scanner with MRI-compatible SiPM sensors.

v FIG. 1:

Coronal torso PET images produced

with different

reconstruction and correction techniques using the same PET

study: (a) Filtered

back projection

(FBP) method; (b) 2D iterative (IT) method (c) 3D IT method with point spread function

(PSF) modelling; (d) 3D IT PSF and time of flight (TOF) method.

The red arrow shows an abnormal focal radio-pharmacon accumulation, which

can be definitely better visualized with the more advanced PET method.

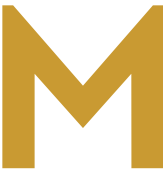
edical diagnosis commonly relies on assessment of both the functional status and the anatomical condition of the patient [1]. In a clinical setting, in-vivo measurement of organ physiology, tissue metabolism, tissue perfusion, and other biological functions can usefully be performed with radionuclide-tracer techniques such as Positron Emission Tomography (PET). However, radionuclide imaging has relatively poor spatial resolution, whereas Magnetic Resonance Imaging (MRI) has excellent spatial resolution and provides rich anatomical details, but yields limited functional content.

The ability to acquire high-resolution anatomical data and as well quantitative functional information in-vivo is becoming increasingly important in the diagnosis and research of diseases. This is clearly reflected in the intention of replacing stand-alone PET by combined PET/MRI methodology as a clinical diagnostic tool, thus combining the advantages of the two. The emission tomography techniques provide functional information of high sensitivity and high specificity while MRI supplies good anatomical detail.

The first human full ring PET scanner was developed in 1975 at Washington University in St. Louis. They used the two annihilation photons that are produced back-toback after positron emission from a radionuclide tagged tracer molecule.

Although the basic detection concept of the two backto-back coincidence photons is still used today, enormous methodological developments have characterized the history of the PET technique. The highly sophisticated image reconstruction, correction and modeling methods allowed achieving an image quality that was beyond expectations. Fig. 1. shows the main steps along that road of development. For each step the year is shown in which the specific method became available in the clinical settings.

The current state of the art in PET/MRI is based on the Avalanche Photo Detector (APD), which has poor
14

\section{The combination of PET/MRI modalities technically is very difficult due to the high magnetic field produced by the MRI which creates a harsh environment for PET electronics $\nabla$}

timing properties ( $>1 \mathrm{~ns}$ ), thus the Time Of Flight (TOF) imaging method (Fig. 1. "d" panel) cannot be applied to this system.

\section{Multi-modality PET/MRI systems}

PET/MRI systems use separate detectors for nuclear magnetic resonance and radionuclide imaging, with the detectors integrated on a common gantry to simplify patient handling, data acquisition, and co-registration of the MRI and PET image data. The MRI and PET data are acquired with the patient in the same position to facilitate image correction. This makes it possible to produce a "fused image" in which the radionuclide distribution can be displayed in colour on top of a grey-scale MRI image to co-register the anatomical and physiological features. This has been shown to dramatically improve diagnosis and evaluation of disease, in comparison to either MRI or PET used alone. Small animal hybrid PET/ MRI system is also an optimal choice for the translational science and for the validation of molecular imaging methods based on these different imaging modalities. However, the combination of these modalities is technically very difficult due to the high magnetic field produced by MRI which creates a harsh environment for PET electronics, mainly for the Photo-Multiplier Tubes (PMT). Another problem is the space constraint,

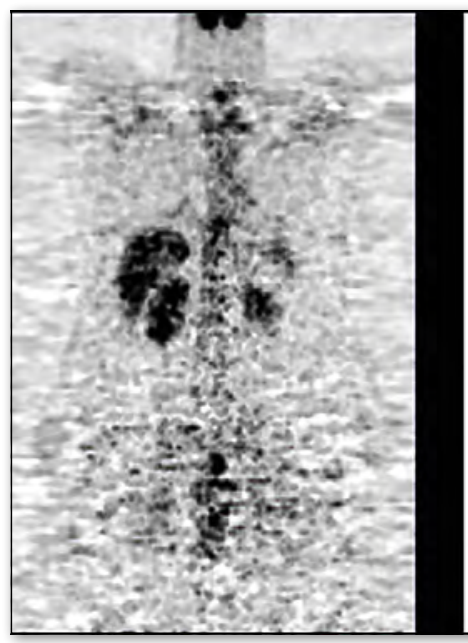

(a) FBP (1975)

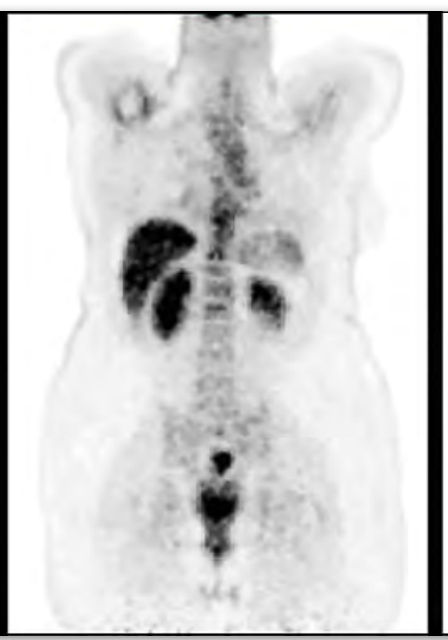

(b) 2D iterative (1997)

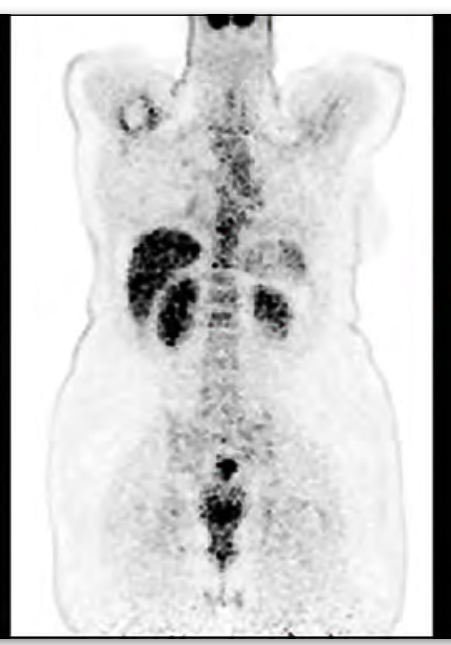

(c) 3D iterative+PSF (2006)

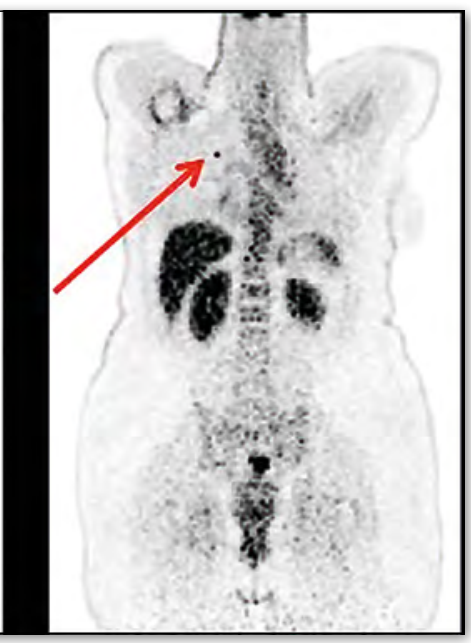

(d) 3D iterative+PSF+TOF (2009) 
because the field of view of MRI is relatively small, making the design of the PET detector inside the MRI bore very challenging. This is a major issue for a small animal PET/MRI camera [2].

The most widely used radiation detector in PET systems is made of a scintillator crystal (Fig. 2) attached to a PMT. The crystal detects the incoming gamma photon and converts its energy into light. This light is detected by the photocathode of the PMT and transformed to a very weak electrical signal. This signal is amplified through an avalanche cascade process in the tube. The use of multi-anode Position-Sensitive Photo-Multiplier Tubes (PSPMT) allows accurate positional and energy information to be derived from the PMT itself [3].

The advantage of this PMT-based detection method is the high gain factor and low noise of the device. The good timing capability is also a benefit. The drawback is the high-voltage requirement of the PMT and its sensitivity to magnetic field.

The rapid developments of the microelectronic industry during the last three decades have yielded high-purity detector-grade silicon, resulting in customized low-noise silicon planar photo-detectors such as Silicon Photo-Multiplier (SiPM) [4]. The current pulses from the avalanche breakdown of each Geiger mode photodiode are summed at the output signal which is proportional to the number of photodiodes breaking down at any particular time. This is in turn proportional to the incident light flux on the sensor. SiPM technology is based on arrays of photon-counting diodes in Geiger mode, each with an integrated quenching circuit and with outputs connected in parallel. The very high gain combined with a high photon detection probability results in a world-record light sensor.

The advantage of the SiPM device is its high gain, high quantum efficiency, low-voltage requirement, fast response and insensitivity to magnetic fields. The limitations are its relatively high price, low fill factor and cell-to-cell optical crosstalk.

\section{MiniPET-3: a SiPM-based small animal PET scanner}

A scanner was developed for molecular imaging of small animals in the framework of the EU project ENIAC-2009-1_120209 Central Nervous System Imaging (CSI). It was manufactured at the Institute for Nuclear Research, Hungarian Academy of Sciences (MTA Atom$\mathrm{ki}$ ), in cooperation with the University of Debrecen, STMicroelectronics and Philips Applied Technologies. The main goal of this project was to demonstrate the applicability of MRI-compatible SiPM sensors for replacing PSPMTs used traditionally for scintillation detectors. The project has been successfully completed with the result of a fully functioning camera shown in Figure p.17.

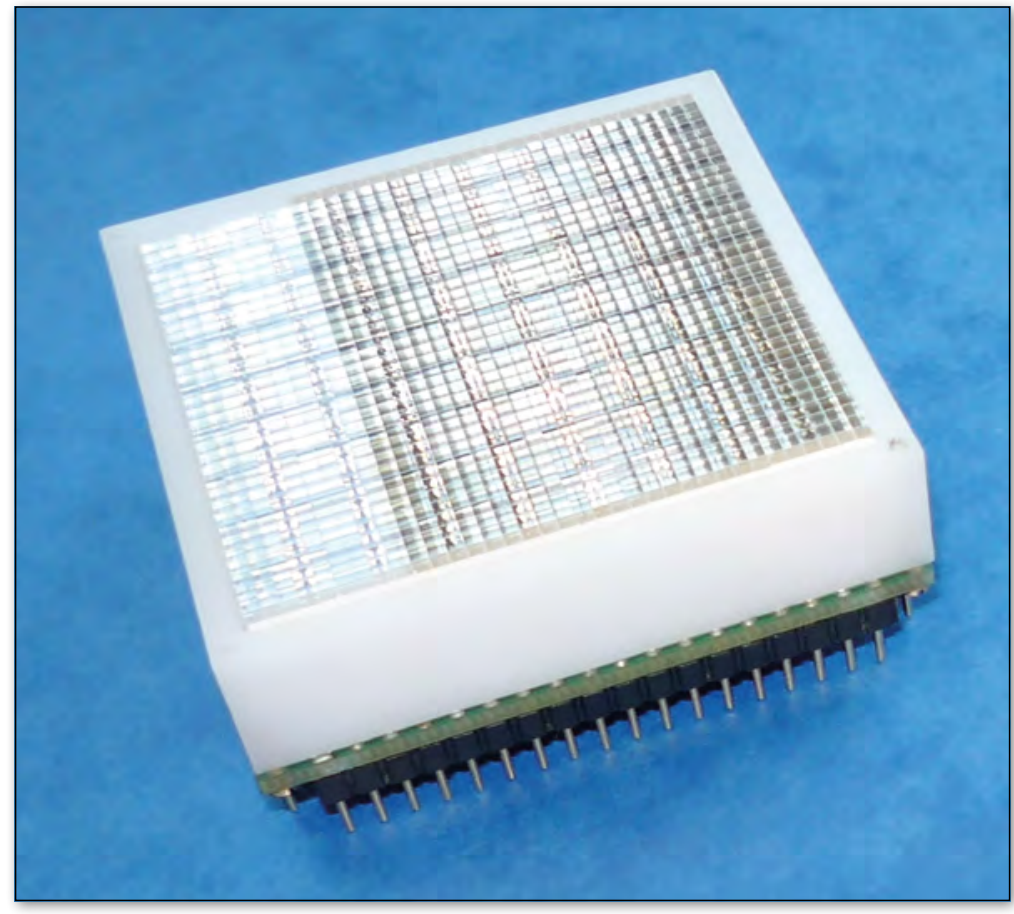

$\triangle F I G .2$ : This Cerium-doped lutetium based scintillation crystal offers high density and short decay time. It is ideal for applications that require better energy resolution and better timing, including PET.

VFIG. 3: Components of the detector module: $35 \times 35$ segmented LYSO crystal matrix, $9 \times 9$ quadpixel element SiPM sensors and analog front-end electronics.

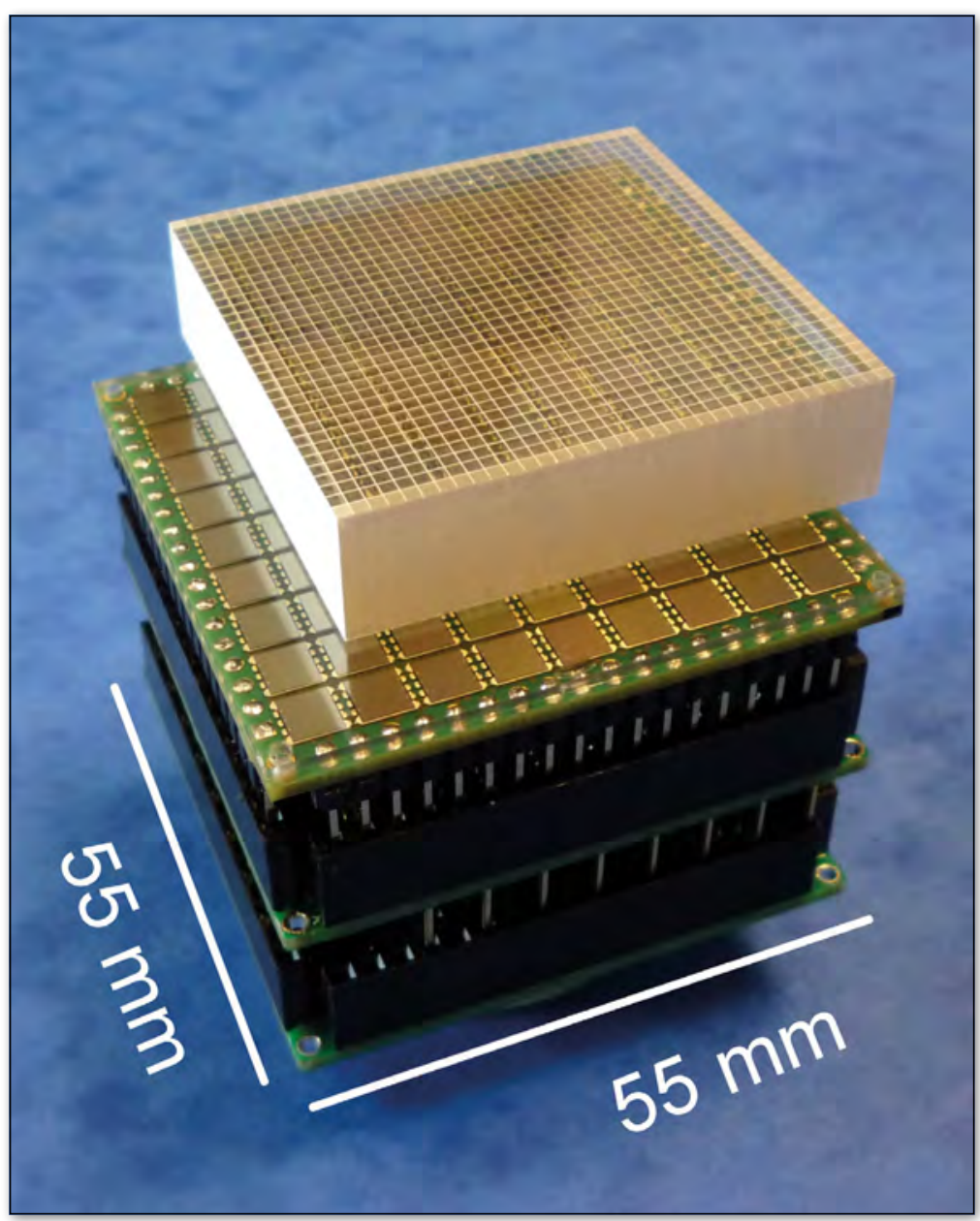




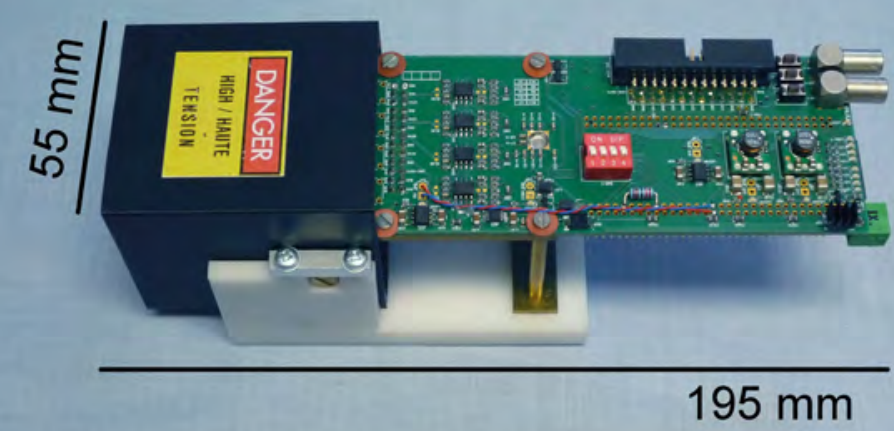

$\triangle$ FIG. 4:

Assembled detector module of the small animal PET scanner. heart measurement after injected FDG radio-pharmacon. The scan time was $15 \mathrm{~min}$.

\section{State-of-the-art technology}

The PET image is constructed via precise determination of the position of electron-positron pair annihilation. The detector is a scintillator matrix, and the points of annihilation gamma impacts are defined by the rows and columns of the matrix. But the SiPM detectors are noisy, which causes deterioration of the readout of a large number of rows and columns operated simultaneously. This problem has been overcome by the use of an additional electronic circuit, which restricts the readout of the matrix to a segment from which a gamma signal is detected.

This solution was successfully used recently in the construction of the first SiPM-based small animal MiniPET-3 scanner, applying an $9 \times 9$ quad-pixel matrix of SiPMs at room temperature coupled to $35 \times 35$ elements LYSO (Lutetium-Yttrium oxyOrthoSilicate) crystal matrix shown in Fig. 3. The total of 36 readout channels was further reduced to four to deliver information on $\mathrm{X}$ and $\mathrm{Y}$ position, energy and time.

The scanner construction is based on identical detector modules, which allows direct replacement of existing units or extension of the system with new units shown in Fig. 4. A list-mode-based data acquisition system of the detectors with a local area network utilizes all the benefits of the widely used standard communication. The coincidence events selection is based on a central timing unit,

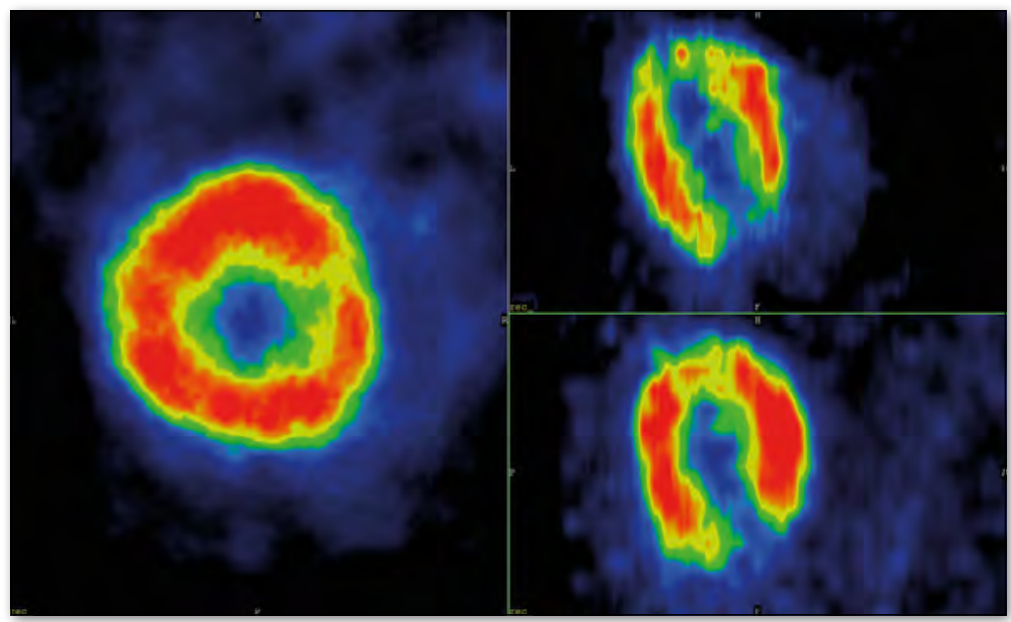

which generates clock signal for precise time-stamping of all events recognized by the detector modules.

The system also contains data acquisition procedures required for calibration, quality control as well as the static, dynamic and whole-body acquisition protocols. Fig. 5. demonstrates the molecular imaging capability of MRI-tolerant MiniPET-3 scanner.

Various applications of tomography have contributed enormously to the development of medical diagnostics. The imaging is based on basic physics: X-ray-, nuclearmagnetic-resonance- or positron-electron pair spectroscopy. They all involve the fast collection and analysis of data arranged into multi-channel arrays, just as the data collected in nuclear and particle physics experiments. Their analysis is also similar to that of physics experiments, and, in complexity, neither is inferior to the other.

\section{Acknowledgements}

This work was supported under the project'Central Nervous System Imaging' by the ENIAC Joint Undertaking (No. 120209) and by the Hungarian National Development Agency (No. ENIAC_08-1-2011-0002)

\section{About the authors}

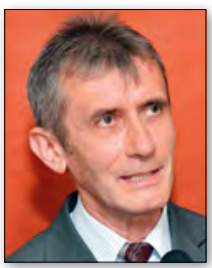

Jozsef Molnar: deputy technical director of Institute for Nuclear Research, Hungarian Academy of Sciences. Graduated at Technical University in Budapest as electronic engineer, Candidate of Technical Sciences at Joint Institute of Nuclear Research, Dubna. Awarded Denes GABOR Prize in 2011.

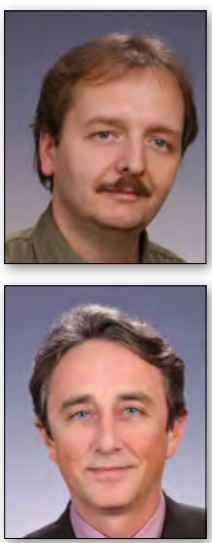

Laszlo Balkay: scientific officer, leader of the medical imaging group at the Department of Nuclear Medicine, University of Debrecen. He graduated at the University of Debrecen as physicist and obtained his $\mathrm{PhD}$ on biophysics.

Ervin Berenyi: full professor, $\mathrm{MD}, \mathrm{PhD}$, head of the Department of BioMedical Laboratory and Imaging Science, University of Debrecen. He graduated at the Medical University of Pécs. He is an expert in the field of radiology and neuroradiology.

\section{References}

[1] Fred A. Mettler, Jr. Milton J. Guiberteau, Essentials of Nuclear Medicine Imaging, $6^{\text {th }}$ Edition, Elsevier (2012).

[2] F. Azaiez et al., Nuclear Physics for Medicine, NuPECC, 59 (2014).

[3] J. Imrek et al., IEEE Nuclear Science Symposium Conference Record 5, 3037 (2006).

[4] J. Imrek et al., IEEE Nuclear Science Symposium Conference Record 0, 3790 (2012).

[5] F. Nagy et al., NIM A 759, 44 (2014). 